\title{
Submonolayer growth of CuPc on noble metal surfaces
}

\author{
Benjamin Stadtmüller,,${ }^{1,2, *}$ Ingo Kröger, ${ }^{1}$ Friedrich Reinert, ${ }^{2,3}$ and Christian Kumpf ${ }^{1}$ \\ ${ }^{1}$ Peter Grünberg Institut (PGI-3), Forschungszentrum Jülich, D-52425 Jülich, Germany, and JARA-Fundamentals of Future \\ Information Technologies (JARA-FIT) \\ ${ }^{2}$ Experimentelle Physik VII, Universität Würzburg, D-97074 Würzburg, Germany \\ ${ }^{3}$ Karlsruhe Institute of Technology (KIT), Gemeinschaftslabor für Nanoanalytik, D-76021 Karlsruhe, Germany
}

(Received 8 October 2010; published 18 February 2011)

\begin{abstract}
The understanding of growth mechanisms and electronic properties is a key issue for improving the performance of small organic devices, in which the metal-organic interface and its properties play a crucial role. In this context we investigated the adsorption behavior and the electronic properties of copper-II-phthalocyanine $(\mathrm{CuPc})$ within the first adsorbate layer on $\mathrm{Au}(111)$ and $\mathrm{Cu}(111)$. Together with recent results published for $\mathrm{CuPc} / \mathrm{Ag}(111)$ [Kröger et al., New J. Phys. 12, 083038 (2010)] this leads to a comprehensive understanding of the adsorption of $\mathrm{CuPc}$ on noble metal surfaces: $\mathrm{On} \mathrm{Cu}(111)$ the molecule-surface interaction is the strongest. The molecules chemisorb on the surface and form one-dimensional chains or two-dimensional islands, depending on coverage. This behavior indicates an attractive intermolecular interaction. In contrast, on $\mathrm{Au}(111) \mathrm{CuPc}$ is only weakly physisorbed and behaves like a two-dimensional gas in a wide coverage regime. Only when densely packed do the molecules form ordered structures, which are scarcely influenced by the structure of the metallic surface. Molecule-molecule interaction is also very weak, but in contrast to $\mathrm{CuPc}$ on $\operatorname{Ag}(111)$ no clear indications for a repulsive interaction are found. Regarding the adsorption strength, this latter system represents an (possibly unique) intermediate case which enables the unusual intermolecular repulsion found recently. Our results highlight the special role of this model system, since the interaction of CuPc with the metal can be "tuned" in any order of the adsorption scenarios observed by selecting the right substrate material.
\end{abstract}

DOI: 10.1103/PhysRevB.83.085416

PACS number(s): 61.05.jh, 79.60.Bm, 74.70.Kn, 68.43.Fg

\section{INTRODUCTION}

The investigation of organic thin films adsorbed on noble metal surfaces is driven by two main goals. The first is the improvement of organic electronic devices such as light emitting diodes, ${ }^{1}$ field effect transistors, ${ }^{2}$ or solar cells. ${ }^{3}$ The second, more fundamental motivation is to obtain a comprehensive understanding of the basic physical properties at metal-organic interfaces. These properties are predominantly influenced by the formation of the very first adsorbate layer, which influences the growth behavior of the entire organic thin film. The key for a fundamental understanding of geometric, electronic, optical, and magnetic properties of metal-organic interfaces is the investigation of the adsorption behavior and structure formation of the molecules in the submonolayer regime. For this reason model systems like 3,4,9,10-perylene-tetra-carboxylic-dianhydride (PTCDA) and other $\pi$-conjugated molecules have been studied in detail for a long time on various surfaces. ${ }^{4-13}$ Also metal-phthalocyanine (MePc) molecules were investigated frequently due to their high thermal stability and high molecular symmetry and since their molecular properties can easily be changed by substituting the central metal atom. Their adsorption on $\mathrm{Cu}(111), \mathrm{Ag}(111)$, and $\mathrm{Au}(111)$ has been studied with various experimental methods. ${ }^{14-21}$ Recent results indicate that MePc molecules show intermolecular repulsion upon adsorption on the $\operatorname{Ag}(111)$ surface, ${ }^{14,15}$ in contrast to the islandlike growth which is usually observed for organic molecules. The $D 4 h$ symmetry of the molecule plays an important role in this context, since it is responsible for vanishing electrostatic dipole and quadrupole moments which would mask intermolecular interactions induced by the substrate. The same is true for hexaperi-hexabenzocoronene (HBC), another highly symmetric molecule for which intermolecular repulsion was reported recently upon adsorption on $\mathrm{Au}(111){ }^{22}$

In the case of $\mathrm{MePc} / \mathrm{Ag}(111)$, the repulsion was explained by donation of electronic charge from the molecule into the surface (partly via the central metal atom) and a backdonation into other parts of the molecule. The resulting molecule-substrate interaction represents a mechanism which is more efficient for diluted molecular layers, i.e., for larger distances between the molecules. ${ }^{14}$ This causes intermolecular repulsion; however, the precise interplay of the charge donation and backdonation is crucial and depends on the molecule-surface interaction. By changing the substrate from $\operatorname{Ag}(111)$ to $\operatorname{Au}(111)$ and $\mathrm{Cu}(111)$ this interaction is altered, weakened for $\mathrm{Au}(111)$ and strengthened for $\mathrm{Cu}(111)$. This motivates the present work in which we systematically studied the geometric and electronic structure of $\mathrm{CuPc}$ on $\mathrm{Au}(111)$ and $\mathrm{Cu}(111)$ within first monolayer.

We present a comprehensive study of the submonolayer growth of $\mathrm{CuPc}$ on $\mathrm{Cu}(111)$ and $\mathrm{Au}(111)$ using high-resolution low-energy electron diffraction and ultraviolet photoelectron spectroscopy at different temperatures. On $\mathrm{Au}(111)$ for low coverages the $\mathrm{CuPc}$ molecules show no ordered structure and behave like a two-dimensional (2D) gas, whereas at higher coverages two long-range-ordered phases occur, a relaxed phase and a more compressed one which represents the monolayer structure. A discrete phase transition from one to the other occurs. Both structures are scarcely influenced by the underlying surface and indicate very weak van der Waals interaction between the molecules and also weak moleculesurface interaction (physisorption). In contrast, on $\mathrm{Cu}(111)$ the $\mathrm{CuPc}$ molecules arrange in linear chains for low coverages, and form well-defined islands of a commensurate structure for high coverages. This indicates an attractive intermolecular 
interaction between the molecules. In addition, a charge transfer from the substrate into the molecule (indicating strong chemisorption) was observed on $\mathrm{Cu}(111)$, but not for $\mathrm{Au}(111)$. In this paper we correlate the different effects in the structure formation with the intermolecular interactions and the binding properties of the molecules at the surface.

\section{EXPERIMENTAL DETAILS}

All measurements were performed using a standard ultrahigh-vacuum system at a base pressure better than $8 \times 10^{-10}$ mbar. The setup consists of a spot profile analysis low-energy electron diffraction (SPA-LEED) instrument, a hemispherical electron analyzer, and photon sources for ultraviolet and $\mathrm{x}$-ray photoemission experiments (UPS and XPS). Sample surfaces [(111)-oriented $\mathrm{Au}$ and $\mathrm{Cu}$ crystals] were cleaned by repeated cycles of Ar ion bombardment $\left( \pm 55^{\circ}\right.$ incident angle of the ion beam, $2 \times 20 \mathrm{~min}, I_{\text {sample }}=4 \mu \mathrm{A}$ ) and subsequent annealing at a temperature of $650^{\circ} \mathrm{C}$ for approx. $40 \mathrm{~min}$. With this preparation cycle very well-ordered surfaces were obtained, indicated by very narrow $(0,0)$-spot profiles. Usually transfer widths of $500 \AA$ were determined for the clean $\mathrm{Au}(111)$ and the $\mathrm{Cu}(111)$ surfaces. The $\mathrm{Au}(111)$ surface also showed sharp diffraction spots of the well known $p \times \sqrt{3}$ herringbone reconstruction, ${ }^{23-25}$ visible up to the third order at least. Its real-space periodicity $p$ was found to be $p=(22 \pm 1)$ which agrees well with literature values. ${ }^{23,24}$ Sublimated $\mathrm{CuPc}$ molecules were deposited on the crystal surfaces using a dedicated evaporator. During deposition the molecular flux was monitored with a quadrupole mass spectrometer and the evaporator temperature was adjusted for a constant deposition rate of $0.5 \mathrm{ML} / \mathrm{min}$ (where ML indicates monolayer). The sample was kept at room temperature during deposition and annealed afterward $\left[280^{\circ} \mathrm{C}\right.$ for $10 \mathrm{~min}$ for $\mathrm{CuPc}$ on $\mathrm{Au}(111)$ and $160^{\circ} \mathrm{C}$ for $20 \mathrm{~min}$ for $\mathrm{CuPc}$ on $\left.\mathrm{Cu}(111)\right]$. The coverage of the molecular layer was obtained from the intensity of the C $1 s$ XPS signal using the signal of the monolayer structure as a reference. The experimental error of this method was determined to be $\pm 0.04 \mathrm{ML}$.

In order to achieve highest $k$-space resolution in the SPA-LEED experiments we chose that section of the LEED images with smallest asymmetric distortions. Then calculated LEED patterns were fitted to the measured data by varying the superstructure matrix entries and manual adjustments. In this way each entry could be determined with an accuracy of \pm 0.025 , which corresponds to an uncertainty of the structural parameters of $\pm 0.10 \AA$ for the length of the unit cell vectors, $\pm 0.40^{\circ}$ for the unit cell angle, and $\pm 1.4 \AA^{2}$ for the area of the unit cell. For the evaluation of intensity profiles all features like spots or diffuse rings were modeled by Gaussian functions, except the global background of the central $(0,0)$ reflection, which was described by a Lorenzian since this represents a better approximation of the thermal diffuse background. $^{26,27}$ For UPS experiments an excitation energy of $21.23 \mathrm{eV}$ was used. The resolution for this experiments was better than $70 \mathrm{meV}$, determined by low-temperature measurements. We also analyzed the spectra of the clean surfaces and especially evaluated the substrate $d$-band satellites caused by the nonmonochromatized photon source. They were modeled by Gaussian peaks with a fixed distance of $1.87 \mathrm{eV}$ and a relative intensity of 0.02 compared to the corresponding $d$ band. This enabled a correct treatment of the background in the vicinity of the photoemission peaks of CuPc molecules.

\section{RESULTS}

In the first part of this section we present the results for $\mathrm{CuPc}$ on $\mathrm{Au}(111)$, and in the second the findings for $\mathrm{CuPc}$ on $\mathrm{Cu}(111)$.

\section{A. CuPc on $\operatorname{Au}(111)$}

The initial growth behavior of $\mathrm{CuPc}$ on $\mathrm{Au}(111)$, which is summarized in the phase diagram in Fig. 1, can be divided in three major sections: The $g$-phase can be found at room temperature for coverages below $0.93 \mathrm{ML}$ and at low temperatures below $0.73 \mathrm{ML}$. In LEED it is characterized by the absence of an ordered diffraction pattern; instead diffuse ring structures are observed. In the range between 0.73 and $0.93 \mathrm{ML}$ a phase transition from the $g$-phase to an ordered low-temperature (LT) phase occurs upon cooling. In contrast, for all coverages above 0.93 ML ordered structures are obtained: A relaxed phase for coverages below 1.00 ML, and a compressed phase, which occurs when the first molecular layer is closed.

\section{1. g-phase}

A typical diffuse LEED pattern that occurs in the range of the $g$-phases (room temperature and coverages below $0.93 \mathrm{ML})$ is shown in Fig. 1. Besides the $(0,0)$ reflection and the gold reconstruction spots, two homogeneous concentric rings are visible. Intensity profiles of these rings for a variety of coverages (see Fig. 2) reveal the different origins of the rings: The outer ring does not change significantly with rising coverage, except for an increase in intensity which just reflects the increasing number of molecules on the surface. The radius of the ring corresponds to a distance of $7.3 \AA$ in real space and remains constant with changing coverage. It is hence due to intramolecular scattering, most likely within the azaporphin ring of the CuPc molecule. ${ }^{14,15}$

The inner ring shows a more interesting behavior: With rising coverage its radius is continuously increasing while

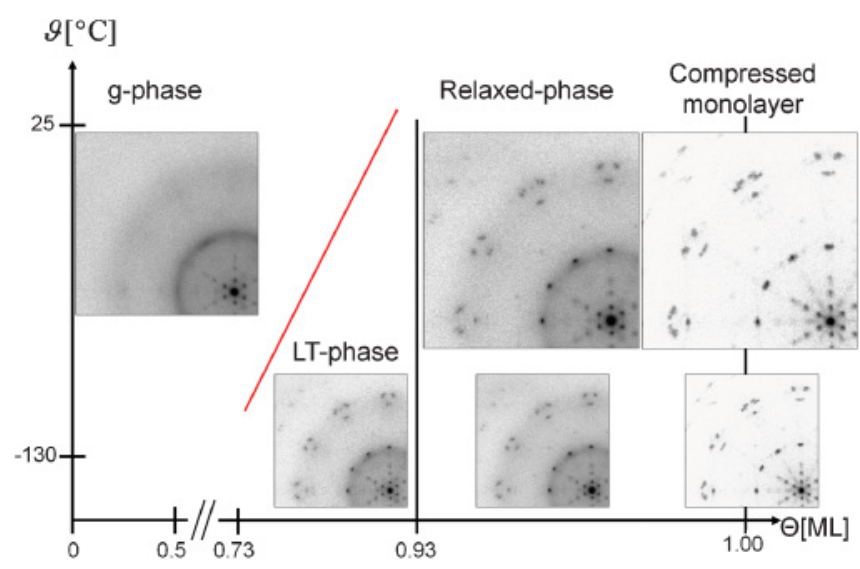

FIG. 1. (Color online) Structural phase diagram for the $\mathrm{CuPc}$ layer on $\mathrm{Au}(111)$ vs temperature $\vartheta$ and coverage $\Theta$ including exemplary LEED pattern for the corresponding phases. 


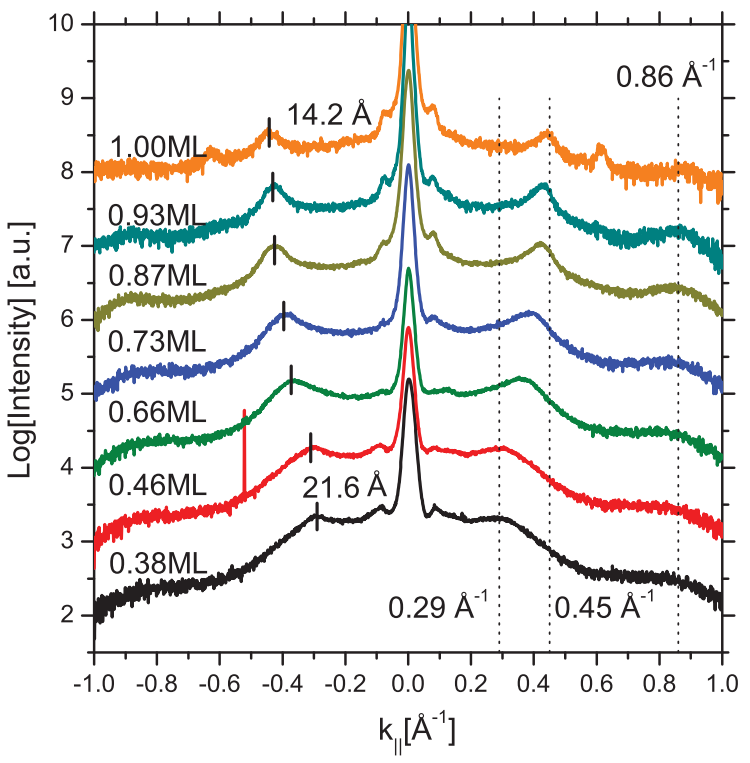

(a)

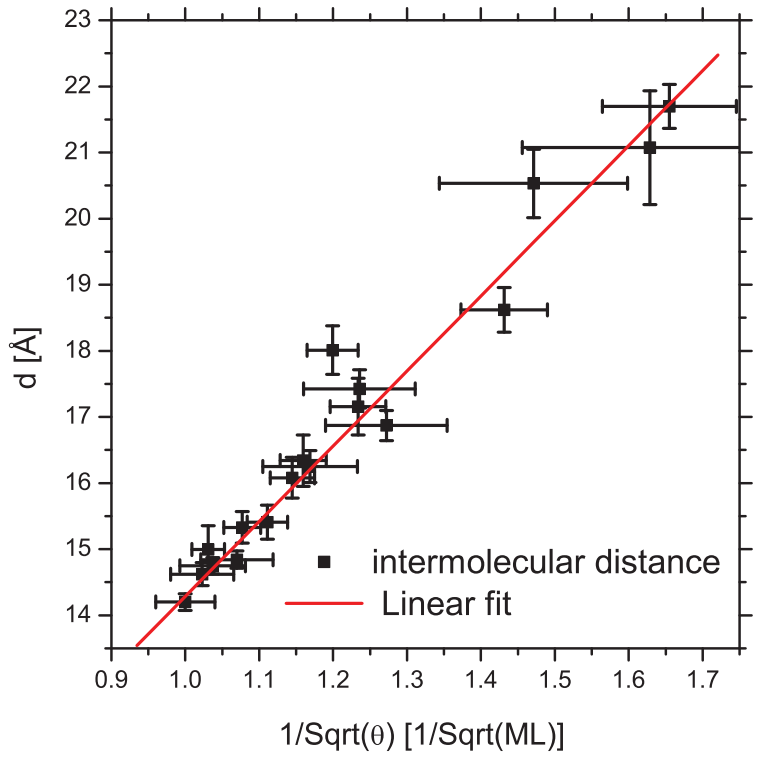

(b)

FIG. 2. (Color online) (a) Intensity profiles of the two diffuse ring structures as a function of coverage; (b) average distance between neighboring molecules as a function of coverage.

its width is decreasing. This indicates that it stems from intermolecular scattering and reflects the average intermolecular distance of the CuPc molecules on the Au surface, which decreases when the disordered layer becomes denser. The intermolecular distances calculated from the ring radii at different coverages agree well with the surface area per molecule calculated from the coverages, assuming that the molecules form the most dilute arrangement on the surface, i.e., the intermolecular distance rises linearly with the square root of the inverse coverage (see Fig. 2). Note that the inverse coverage corresponds to the surface area per molecule. This finding is similar to the behavior of SnPc (Ref. 14) and $\mathrm{CuPc}$ (Ref. 15) on $\operatorname{Ag}(111)$. Furthermore, the sharpening of the ring structure at higher coverages indicates a narrowing of the distribution of intermolecular distances. This is in agreement with the fact that at higher coverages the molecules have less space for (thermally activated) movements since their average next-neighbor distance approaches the size of the molecules. [At a coverage of $0.90 \mathrm{ML}$ the intermolecular distance calculated from the ring radius is $\approx 14.8 \AA$ whereas the diameter of the molecule (van der Waals radiius) is $\approx 14.9$ A.] It should also be mentioned that no second order of this diffuse intermolecular scattering ring is visible, and that the azimuthal intensity distribution in the ring is very homogeneous, which indicates a randomly distributed polar alignment of the molecules. In conclusion [and in analogy to SnPc (Ref. 14) and CuPc (Ref. 15) on $\mathrm{Ag}(111)$ ], we can state that the $\mathrm{CuPc}$ molecules on $\mathrm{Au}(111)$ behave like a $2 \mathrm{D}$ gas. At low temperatures $\left(\approx-130^{\circ} \mathrm{C}\right)$ and for coverages below $0.73 \mathrm{ML}$, we also observed only a diffuse LEED pattern. Below $0.50 \mathrm{ML}$ the diffraction features even remain completely unchanged upon cooling. For higher coverages (between 0.50 and $0.73 \mathrm{ML}$ ), however, the average intermolecular distance increases upon cooling and the intramolecular scattering ring shows a 12-fold azimuthal modulation with maxima in the directions parallel and perpendicular to the $\mathrm{Au}(111)$ highsymmetry axes. While the ring modulation can be explained by an alignment of the molecular wings along the [110], [10 $\overline{1}]$, and $[0 \overline{1} 1]$ substrate directions, ${ }^{15}$ the change in intermolecular distance is probably caused by a condensation of some of the molecules at corrugation lines of the Au surface reconstruction. This causes an increase of the surface area for the remaining molecules and hence the average distance between these molecules increases. This interpretation is consistent with scanning tunneling microscopy (STM) images obtained by Cheng et al., ${ }^{28}$ which show that phthalocyanine molecules favor an arrangement along reconstruction lines at low temperatures. The preferred orientation of the molecular wings along the substrate directions mentioned above was also found in STM. ${ }^{28,29}$

\section{Low-temperature phase}

In the coverage regime between 0.73 and $0.93 \mathrm{ML}$ a reversible phase transition from the diffuse gaslike phase as described above to an ordered low-temperature phase was observed. The latter will be referred to as the "LT phase". A corresponding LEED pattern is shown in Fig. 3(a) and turns out to be of point-on-line type ${ }^{30,31}$ with respect to the uppermost Au layer. Note that-compared to the bulk layersthis uppermost Au layer itself is uniaxially compressed along the $[\overline{1} 10]$ direction. ${ }^{24}$ Furthermore, the reconstruction of the uppermost $\mathrm{Au}$ layer is altered by the adsorption of the molecules (see below). Besides the ordered structure there is still a weak diffuse ring visible in the LEED image, which indicates that a part of the molecular layer remains disordered, i.e., the LT phase is a mixed phase.

The phase transition can be understood in more detail from radial intensity cuts through the intermolecular scattering ring and the superstructure spots which are shown in Fig. 3(b) for 


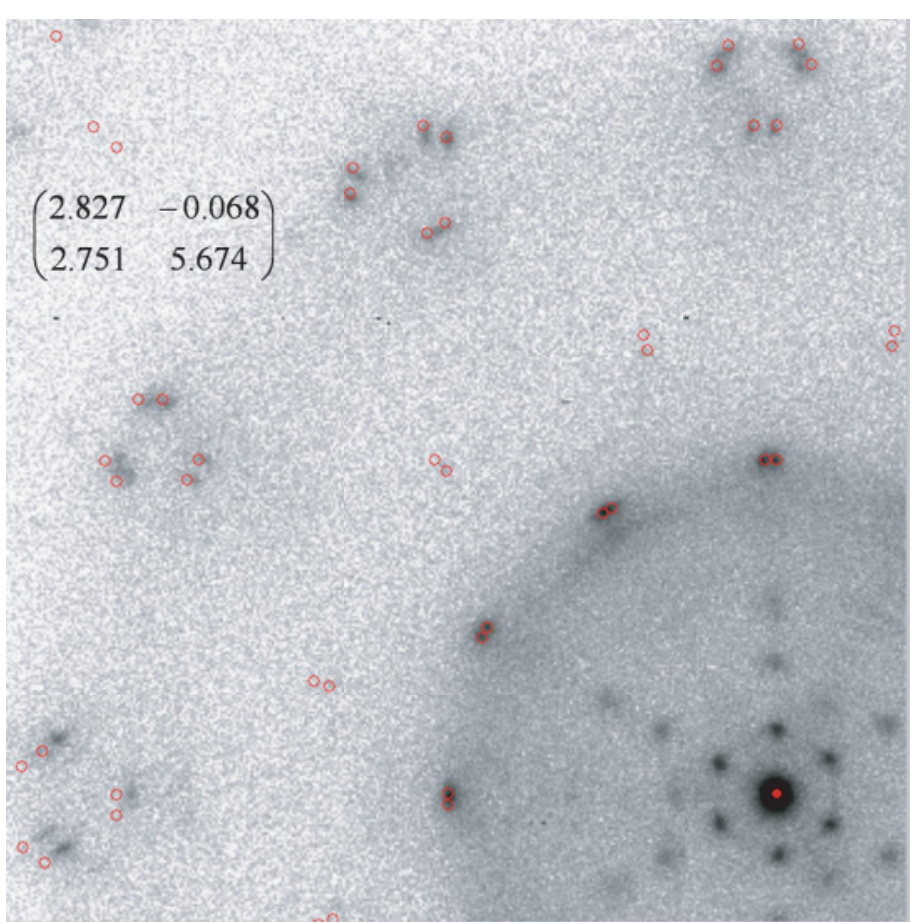

(a)
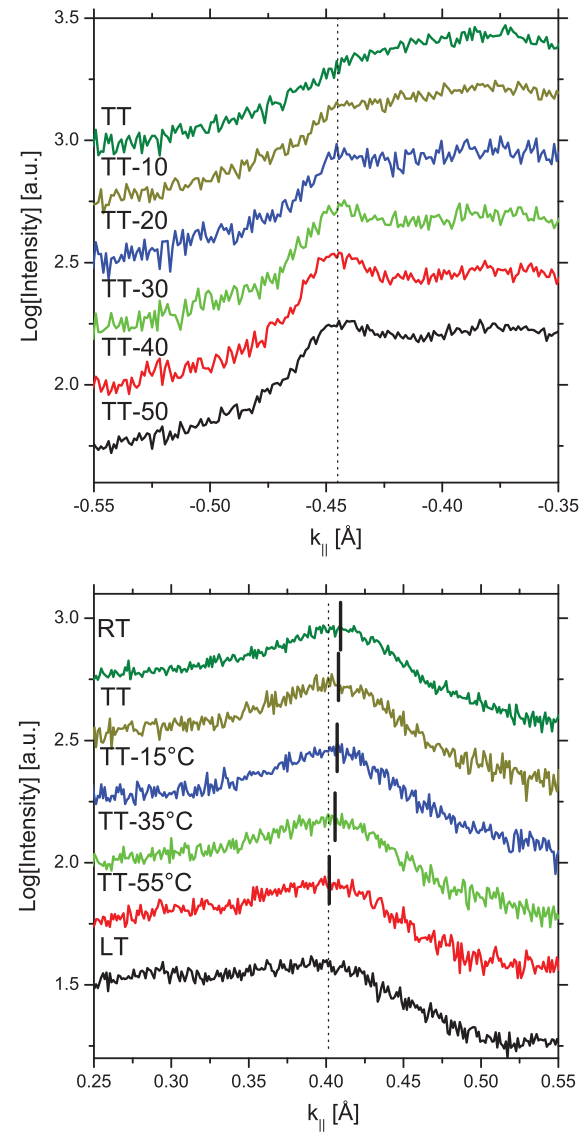

(b)

FIG. 3. (Color online) (a) LEED pattern of the LT phase for $\mathrm{CuPc} / \mathrm{Au}(111)$ recorded at $-130{ }^{\circ} \mathrm{C}$ and an electron energy of $27.2 \mathrm{eV}$. (b) Intensity cuts through diffraction spots (upper part) and through the diffuse ring structure (lower part). The profiles were recorded at decreasing temperatures beginning at the transition temperature (TT).

different temperatures at and below the transition temperature. With rising temperature the intensities of the superstructure spots decrease until the diffraction maxima vanish at the transition temperature. In parallel, the peak in the intensity cuts through the ring shifts slightly to higher $q$ values, indicating a decreasing intermolecular distance. This behavior indicates that the phase transition is basically a condensation of molecules in ordered islands caused by a reduced mobility of the molecules at LT. The formation of such a point-on-line superstructure hence represents the transition to the ground state of the system caused by the minimum in the total interface potential. ${ }^{18}$ However, in contrast to the silver surface, on which MePc molecules form commensurate LT structures in this coverage regime, ${ }^{14,15,17}$ the system under study "only" arranges itself in a point-on-line structure. This emphasizes that the interaction of $\mathrm{CuPc}$ with the gold surface is weaker than that with the $\operatorname{Ag}(111)$ surface.

\section{Relaxed phase and compressed monolayer structures}

All structures between 0.93 and 1.00 ML (excluding the monolayer structure) represent relaxed phases. Several slightly different LEED patterns of well-ordered structures were found in this range. They are all very similar to the LT phase [see Fig. 3(a)]. The data shown in the three panels of Fig. 4(a) demonstrate that the unit cell is slightly shrinking when the coverage is increased. This trend is detectable, even though the change is still just within the error bar of each individual measurement. Note that we plotted the unit cell parameters and size versus the intermolecular distance (obtained from the maximum of the diffuse ring), since an XPS-based coverage determination resulted in much larger error bars. In the right part of the figure the unit cell vectors of relaxed structures with the lowest (blue) and highest (red) coverage are displayed on the grid of the uppermost substrate layer. From the circles in the enlarged details, which show the precise positions of the heads of the corresponding unit cell vectors (the circle represents the experimental error), it can be seen that all relaxed structures are point-on-line. The area of the unit cell is reduced from (197 \pm 1.4) to $(194 \pm 1.4) \AA^{2}$ when the coverage changes from 0.93 to $1.00 \mathrm{ML}$. This corresponds to a relative change of area per molecule of about $2 \%$. The effect is hence much smaller than for $\mathrm{CuPc}$ on $\mathrm{Ag}(111)$, where a continuous compression of the area per molecule of about $12 \%$ was reported in approximately the same coverage range. ${ }^{15}$

Increasing the coverage to one monolayer leads to two coexisting structures which in the following will be referred to as structures $\alpha_{1}$ and $\alpha_{2}$. Their LEED images differ clearly from all other structures observed on the Au surface, in particular in the arrangement of the second-order diffraction spots. Just 

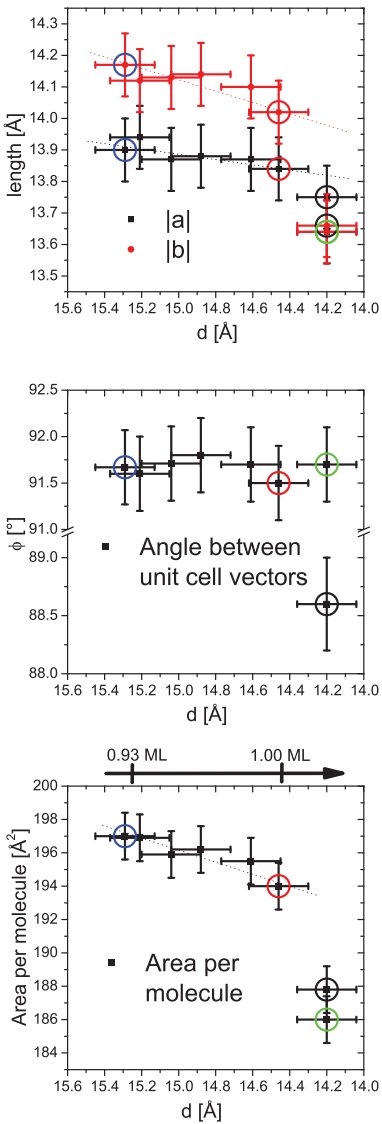

(a)
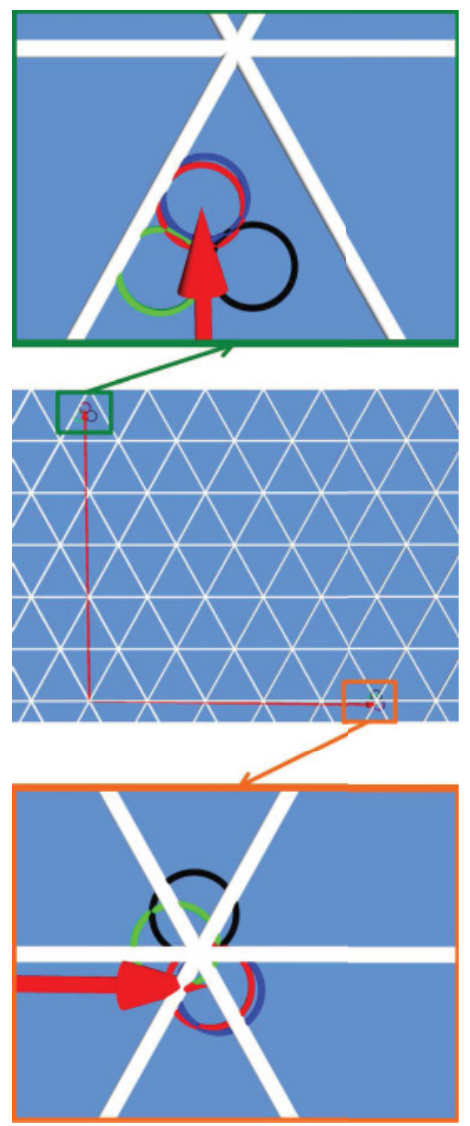

(b)

FIG. 4. (Color online) (a) Parameters of the unit cells found for the relaxed phases (0.93-1.00 ML) and the monolayer structure plotted versus the average intermolecular distance. (b) Corresponding unit cells drawn on the surface lattice of the reconstructed $\mathrm{Au}(111)$. Circles indicate positions and errors of the unit cell vectors. Blue and red circles refer to the relaxed phases with lowest and highest coverage, respectively, green to the monolayer $\alpha_{1}$, and black to the monolayer $\alpha_{2}$ structure.

like all other relaxed structures, these monolayer structures also do not change upon cooling. In Fig. 4 the unit cells of both compressed monolayer structures are shown in green $\left(\alpha_{1}\right)$ and black $\left(\alpha_{2}\right)$. Within the experimental error $\alpha_{1}$ can be characterized as point-on-line; $\alpha_{2}$ is incommensurate. The area per molecule is $4 \%$ smaller than that of the relaxed structures, which indicates a noncontinuous transition from the relaxed to the compressed phase when the coverage is increased.

Although for submonolayer coverages close to $1.00 \mathrm{ML}$ the structures on $\mathrm{Au}(111)$, as well as on $\mathrm{Ag}(111),{ }^{15}$ can be classified as point-on-line, there are significant differences in the molecular order on the different surfaces. For weakly interacting systems like PTCDA on Au(111) (Ref. 32) and highly oriented pyrolytic graphite ${ }^{31}$ (HOPG) or TiOPc on Au(111), ${ }^{18}$ point-on-line structures are frequently observed. This is also the case for the system under study, $\mathrm{CuPc}$ on $\mathrm{Au}(111)$, where the superstructures are slightly changing, which results in a marginal decrease of area per molecule as a function of coverage. This change of structural parameters is a result of the minimization of the total potential energy of the interface when more molecules are adsorbing on the surface, a finding which is supported by Lennard-Jones-based potential calculations ${ }^{18}$ for the TiOPc/Au(111) interface, where the optimized monolayer structure is of point-on-line type. However, the shrinking effect of the unit cell is much smaller compared to $\mathrm{CuPc}$ and $\mathrm{SnPc}$ on $\operatorname{Ag}(111)$, and therefore it cannot be seen as a proof for repulsive interaction between the Pc molecules. On the other hand there are also no clear signs for the formation of islands on the surface at lower coverage, which would have proven attractive intermolecular interaction. Instead a discrete phase transition to the monolayer phases occurs only at very high coverages close to one monolayer. This indicates that the molecules are only very weakly interacting with each other and that only sterical circumstances force the layer to the formation of relaxed and compressed phases at high coverages.

\section{The $22 \times \sqrt{3}$ reconstruction}

All recorded LEED patterns still show the typical spots of the $22 \times \sqrt{3}$ surface reconstruction of the $\mathrm{Au}(111)$ surface which indicates that the reconstruction is not lifted by the adsorption of CuPc. This is in agreement with STM images of the monolayer structure of TiOPc on $\mathrm{Au}(111),{ }^{18}$ where the zigzag pattern of the Au reconstruction is visible through the first molecular layer. However, radial intensity profiles through the first-order reconstruction spots reveal a continuous change of the periodicity with increasing coverage. It should be mentioned that this change occurs only after annealing the adsorbate layer (at $280^{\circ} \mathrm{C}$ in our case), whereas directly after adsorption (at room temperature) the original $22 \times \sqrt{3}$ reconstruction remains unchanged. After annealing, the periodicity $p$ of the $p \times \sqrt{3}$ reconstruction depends on the $\mathrm{CuPc}$ coverage. As illustrated in Fig. 5, $p$ increases almost linearly from $22 \pm 1$ (clean surface) to $26 \pm 1$ (coverage of $1 \mathrm{ML}$ ). A similar change was found for the monolayer structures of other organic molecules like $\mathrm{C}_{60},{ }^{33} \mathrm{PTCDA},{ }^{5} \mathrm{HBC},{ }^{34}$ and even TiOPc. ${ }^{18}$ The increased reconstruction periodicity indicates a reduction of surface stress along the [110] direction in the uppermost Au layer, which is as large as $(18 \pm 6) \%$ and needs to be activated by annealing the system.

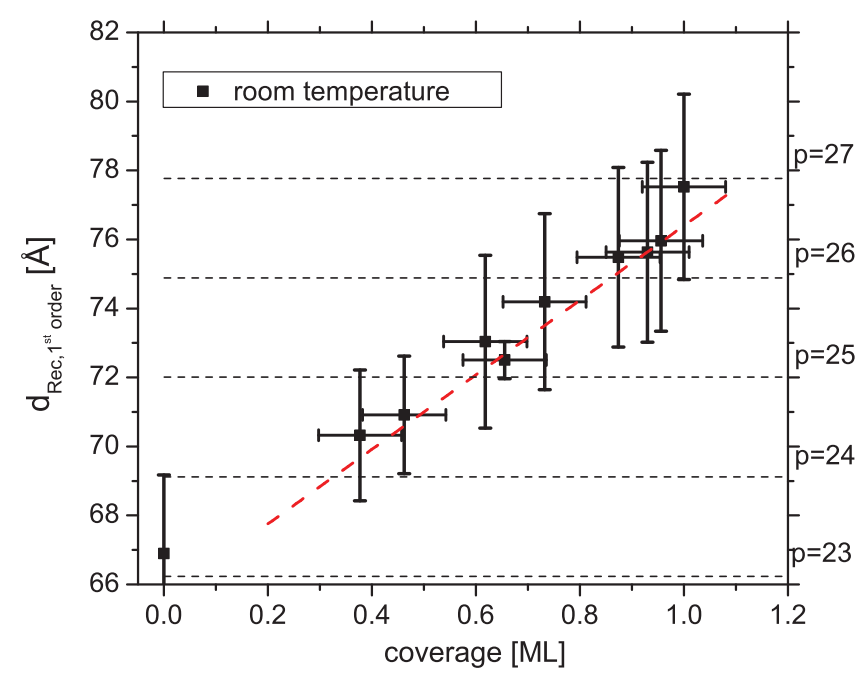

FIG. 5. (Color online) Periodicity of the $\mathrm{Au}(111)$ surface herringbone reconstruction in [110] direction determined by the positions of first-order diffraction spots. 


\section{Electronic structure of the CuPc/Au(111) interface}

In Fig. 6 the electronic valence structure of the CuPc films on $\mathrm{Au}(111)$ measured at an emission angle of $45^{\circ}$ is shown for different coverages. This emission angle was chosen in order to maximize the photoelectron yield. ${ }^{35}$ The submonolayer and monolayer spectra were taken at $\mathrm{LT}=-130^{\circ} \mathrm{C}$, the clean surface and multilayer spectra at room temperature. All spectra (except that of the clean surface) clearly show a peak at low binding energy $(0.6-1.0 \mathrm{eV})$ which can be assigned to the highest occupied molecular orbital (HOMO) of the $\mathrm{CuPc}$ molecule. The asymmetric shape of the peak is a result of the vibronic progression and well known from gas phase measurements ${ }^{36}$ and experiments on HOPG. ${ }^{37}$ In contrast to results reported for phthalocyanine molecules on other surfaces like $\operatorname{Ag}(111),{ }^{15,16}$ no peak is visible directly at the Fermi edge, i.e., there is no state cutting the Fermi level. This indicates that no considerable charge transfer into

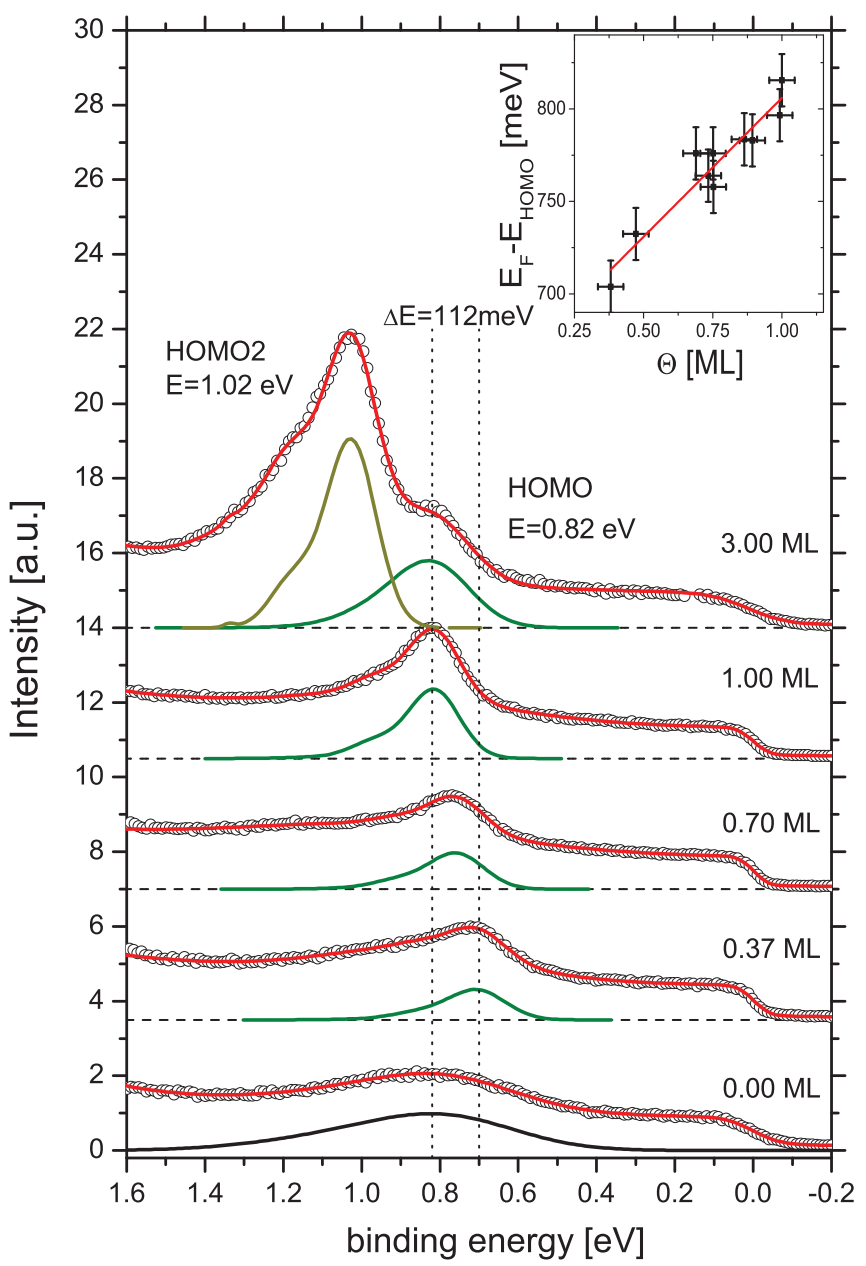

FIG. 6. (Color online) UPS valence spectra of the $\mathrm{CuPc} / \mathrm{Au}(111)$ interface for different coverages measured at different temperatures (submonolayer and monolayer at $\mathrm{LT}=-130^{\circ} \mathrm{C}$ and clean surface and multilayer at room temperature) and an emission angle of $45^{\circ}$. In addition to measured spectra, fitted curves for the HOMO orbital are also shown. Inset: HOMO binding energy as a function of coverage. Note that the density of states around $0.8 \mathrm{eV}$ for clean $\mathrm{Au}(111)$ can be assigned to a $d$-band satellite due to our nonmonochromatized ultraviolet source. the lowest unoccupied molecular orbital (LUMO) takes place. From this fact we can conclude directly that the molecules interact only weakly with the surface; they are physisorbed. For increasing coverages the HOMO state shifts from $0.71 \mathrm{eV}$ at $0.37 \mathrm{ML}$ to $0.82 \mathrm{eV}$ at $1.00 \mathrm{ML}$. A closer investigation reveals that the $(112 \pm 28) \mathrm{meV}$ shift is a linear function of the coverage, i.e., the shift is linearly correlated with the area per molecule on the surface. This can be understood as a local work function effect. The local work function in the vicinity of a physisorbed molecule is reduced by the pushback of metallic electron density due to the presence of the molecule. Since there is no chemical interaction between surface and molecule, the molecular electronic states are pinned to the local vacuum level, not to the Fermi level, and hence the decrease in local work function will also shift the electronic states with respect to the Fermi level. This explains the shift of the HOMO state which we observed for CuPc on $\mathrm{Au}(111)$.

Increase in the coverage beyond 1.0 ML (a spectrum for 3.0 ML is shown in Fig. 6) leads to a splitting of the peak into two components, one located at the position of the monolayer HOMO, the other shifted by $(205 \pm 28) \mathrm{meV}$ to higher binding energy. The first is the remaining signal from the molecular layer in direct contact with the Ag surface. The latter peak represents the second- (and higher-) layer component and is shifted due to a different screening mechanism of the photohole created during the photoionization process, which is caused by the different surroundings of the molecules. Furthermore the polarization of the molecules depends on the layer thickness and also causes an energy shift of the occupied orbitals as observed for rare gas atoms on metal surfaces. ${ }^{38}$

\section{B. $\mathrm{CuPc}$ on $\mathrm{Cu}(111)$}

At first sight the phase diagram obtained for the submonolayer growth of $\mathrm{CuPc}$ on $\mathrm{Cu}(111)$ (see Fig. 7) is much simpler than the one for $\mathrm{CuPc}$ on $\mathrm{Au}(111)$. For coverages below $0.76 \mathrm{ML}$ only diffuse LEED patterns with some rings were observed, indicating a disordered diluted molecular layer ( $d$-phase). For all higher coverages the molecules order in islands having a commensurate structure. Increase in the

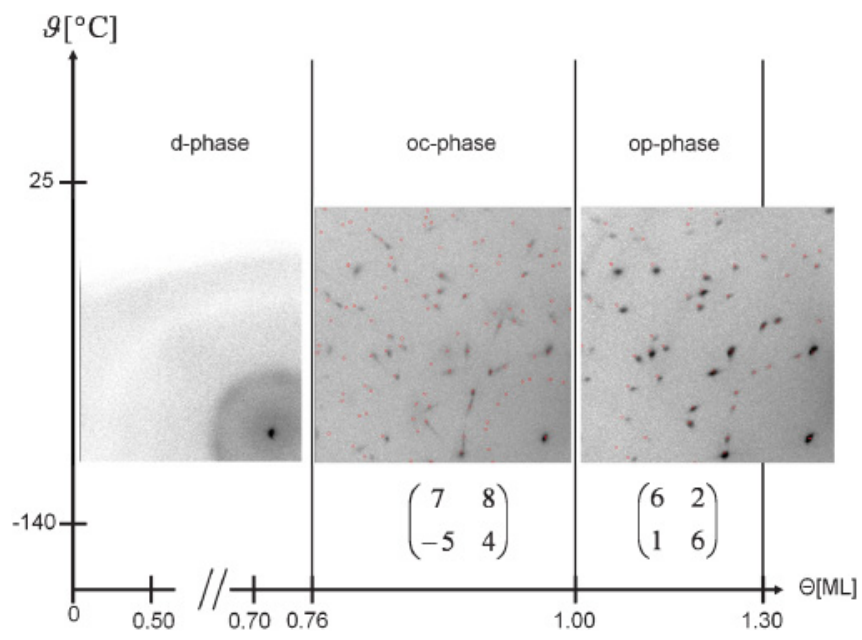

FIG. 7. (Color online) Structural phase diagram for the $\mathrm{CuPc}$ layer on $\mathrm{Cu}(111)$ vs temperature $\vartheta$ and coverage $\Theta$ including exemplary LEED pattern for the corresponding phases. 
coverage changes the spot intensity, but not the spot position. This shows that the periodicity of the molecular islands remains unchanged while the islands are growing.

\section{1. d-phase}

Similar to the results found for $\mathrm{CuPc}$ on $\mathrm{Au}(111)$ in this work and on $\operatorname{Ag}(111),{ }^{15}$ the molecules show no long-range order for low coverages on the $\mathrm{Cu}(111)$ surface. However, a closer look at the intensity profiles reveals distinct differences between these systems [see Fig. 8(a)]. While the radius of the inner ring (representing intermolecular scattering, i.e., the distance between the molecules) is increasing with rising coverage (as expected, since the layer becomes more densely packed) its intensity is decreasing despite the increasing number of molecules on the surface. In order to understand this effect we recorded the ring profiles for each coverage also at different electron energies [see Fig. 8(b)]. It turned out that the ring intensity [normalized to the intensity of the (00) spot] shows a distinct minimum at a certain electron energy, and that this minimum shifts to higher energies when the coverage is increased. Such a minimum at a certain electron energy is caused by a destructive phase shift between the electrons scattered at the surface and at the adsorbate, ${ }^{39}$ and therefore depends on the adsorption height of the molecules. Hence, a change in the adsorption height also shifts the energetic position of the minimum intensity of the LEED spots and can be responsible for the intensity decrease which we observed. One can even use the shift of the minimum to roughly estimate the change in the adsorption height of the molecules with
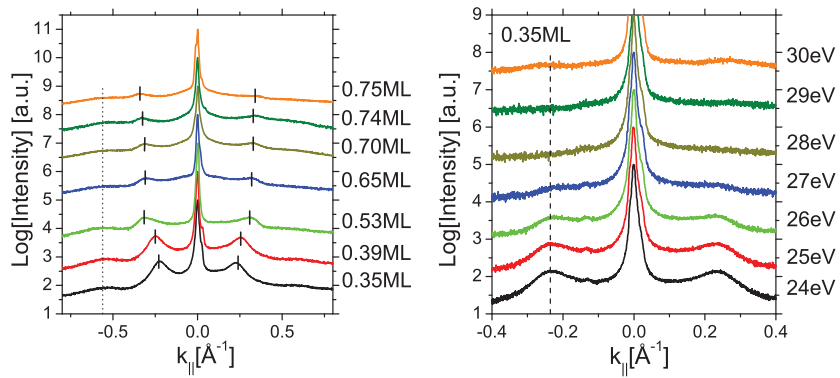

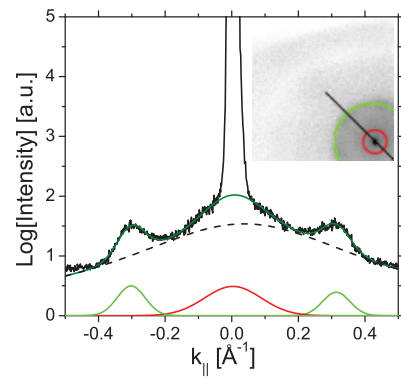

(a)

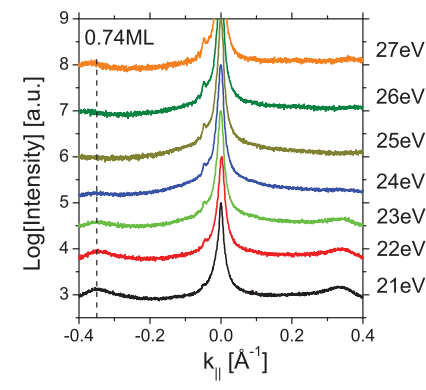

(b)
FIG. 8. (Color online) (a) Upper part: Radial intensity profiles of the ring structures for different coverages measured at $23 \mathrm{eV}$; (a) lower part: fit of the ring intensity encircling the $(0,0)$ spot (red curve) and the intermolecular scattering ring (green curves, intensity profile measured at $23 \mathrm{eV}$ ). (b) Energy intensity profile series for $0.35 \mathrm{ML}$ (upper part) and 0.74 ML (lower part). increasing coverage. One obtains an increase of $\approx 0.5 \AA / \mathrm{ML}$. Even though this value is only a rough estimate it shows the same tendency (i.e., a higher absorption distance with rising coverage) that was observed for CuPc on $\mathrm{Ag}(111)$ using the $\mathrm{X}$-ray standing wave (XSW) technique. ${ }^{15}$ Furthermore, the intermolecular scattering ring shows a modulation with high intensities in the [ $\overline{1} 10]$ and [ $\overline{1} \overline{1} 2]$ directions of the substrate. The modulation indicates a nonhomogeneous distribution of the molecules on the surface, in contrast to the findings on $\mathrm{Au}(111)$ and $\mathrm{Ag}(111)$. The molecules preferably align themselves along these substrate directions. Intermolecular distances obtained from the ring radius [using the line scans in Fig. 8(a)] show a different coverage dependence than on gold and silver. ${ }^{15}$ Above $0.50 \mathrm{ML}$ the intermolecular distances for $\mathrm{CuPc}$ on $\mathrm{Cu}$ are larger compared to the values found for $\mathrm{Au}$ and $\mathrm{Ag}$.

For $\mathrm{CuPc} / \mathrm{Cu}(111)$ and coverages above $0.50 \mathrm{ML}$ an additional intensity ring is visible, closely encircling the $(0,0)$ reflection. This ring was not found for $\mathrm{CuPc}$ on the $\mathrm{Ag}$ and $\mathrm{Au}$ substrates. It indicates that the adsorbates order in islands of random size and distance. ${ }^{40}$ The full width at half maximum of this circle gives the characteristic length of island size and distance. We modeled the circle intensity with a Gaussian function [red curve in Fig. 8(a) lower part] and obtained a mean island size of $\approx 40 \AA$, which does not change significantly with coverage.

Combining the experimental findings for this coverage regime the following growth scenario model can be obtained: For coverages below $0.50 \mathrm{ML}$ the intermolecular distance is too large for a significant clustering of the molecules in islands or chains. The mobility of the molecules is small, so a diluted disordered molecular layer is formed on the surface. When the coverage rises the molecules start to cluster and (as can be seen in STM images; see Ref. 17) form linear chains rather than islands. Since molecules arranged in linear chains are not homogeneously distributed, this growth mode could also explain the modulated intensity of the intermolecular scattering ring. The "island size" discussed above possibly reflects the length of these chains rather than the size of twodimensional islands. At higher coverage also island formation starts, as discussed in the following.

\section{Orthorhombic primitive (op) and centered (oc) monolayer phases}

At coverages above $0.76 \mathrm{ML}$ the molecules start to form islands with a commensurate monolayer structure. Its superstructure matrix as well as the corresponding LEED pattern are displayed in the phase diagram in Fig. 7. The large unit cell size of $(385.9 \pm 1.4) \AA^{2}$ indicates that there are two molecules per unit cell. Analyzing the spot intensities reveals that all spots with one even and one odd index are almost extinguished. This indicates a centered unit cell whereby the two molecular scatterers differ only slightly. Possibly they have slightly different adsorption heights (caused by different adsorption sites), different polar alignments, or are differently bent. Due to its almost orthorhombic centered unit cell we entitle this phase the "oc phase" in the following.

When the coverage is increased from 0.76 to $1.30 \mathrm{ML}$ the intensities of the weak spots mentioned above decrease further 


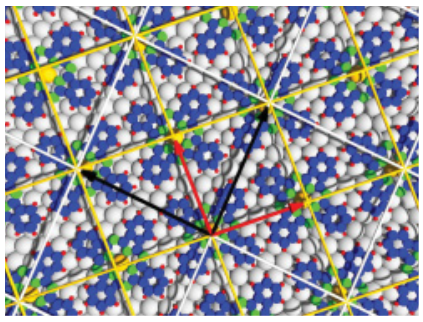

(a)

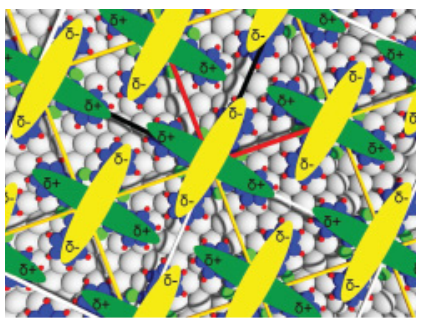

(b)
FIG. 9. (Color online) (a) Real-space model of the commensurate superstructure lattices found for $\mathrm{CuPc} / \mathrm{Cu}(111)$. The unit cells of the oc and op structures are indicated by a white lattice with black unit cell vectors and a yellow lattice with red vectors, respectively. (b) Partial charging of the molecule caused by the charge transfer with the surface.

until they completely vanish. The intensities of other spots also change, but not their positions. The remaining LEED spots can be indexed by the superstructure matrix $(6,2 \mid 1,6)$ which describes a primitive unit cell of exactly half the size of the oc phase. This phase is called the "op phase" in the following. The real-space model shown in Fig. 9(a) suggests that the molecules are aligned along substrate lines which was also found in recent STM images. ${ }^{17}$ Furthermore, the STM study showed that the fourfold symmetry of the CuPc molecules on $\mathrm{Cu}(111)$ is reduced to a twofold symmetry caused by partially filling the degenerated LUMO state (see Sec. III B 3). This symmetry breaking caused by an asymmetric charge distribution in the molecule goes along with an induced electrostatic quadrupole moment on the molecule, and hence influences the intermolecular interaction. The reason for the asymmetric charge distribution (which in the end lifts the degeneracy of the two LUMO states) lies in the different symmetry of substrate and molecules. Since a fourfold symmetric molecule adsorbs on a threefold substrate the four "wings" of the molecules have pairwise a different registry with the substrate atoms. This gives rise to a different charge transfer of neighboring wings of a molecule, whereas opposing wings have identical situations. The consequence is the breaking of the fourfold molecular symmetry and the induced quadrupole moment discussed here.

Since the unit cell in the oc phase is not exactly rectangular, a slight tilt of the centered molecule reduces the electrostatic potential of the layer and leads to the fact that a larger unit cell containing two nonequivalent molecules is energetically favorable. When the coverage is increased further the quadrupole moment obviously becomes screened and the described effect is reduced. This lets the centered molecule align itself more like the other molecule and reduces the intensity of the corresponding LEED diffraction spots until they vanish completely when the unit cell is perfectly centered. The screening of the induced quadrupole moment might be caused by the adsorption of molecules in the second layer, since we annealed the layer only at temperatures far below the second-layer desorption temperature for $\mathrm{CuPc}$ in order to avoid damage to the first layer.

In conclusion, the island growth of $\mathrm{CuPc}$ on $\mathrm{Cu}(111)$ occurring at high submonolayer coverages is a proof of an overall attractive interaction between the molecules. The attractive interaction can be explained by an induced quadrupole moment on the molecules, and is in contrast to the repulsive intermolecular interaction found for CuPc (Ref. 15) and SnPc (Ref. 14) on $\operatorname{Ag}(111)$. The appearance of commensurate structures also proves a stronger interaction between the adsorbate molecules and the $\mathrm{Cu}(111)$ substrate (compared to $\mathrm{Ag})$.

\section{Electronic structure of the $\mathrm{CuPc} / \mathrm{Cu}(111)$ interface}

Figure 10 shows a photoemission spectrum of the vicinity of the Fermi edge for $T=-140^{\circ} \mathrm{C}$ and for a coverage of $0.85 \mathrm{ML} \mathrm{CuPc}$ on $\mathrm{Cu}(111)$. Again an emission angle of $45^{\circ}$ was chosen since the intensity of the molecular states is largest for high emission angles. ${ }^{35}$ In addition to the HOMO peak at a binding energy of approx. $1.5 \mathrm{eV}$ a second broad peak can be seen which is cut by the Fermi edge. It represents the former LUMO (F-LUMO) state and is now partially filled, indicating a chemical interaction between the molecules and the surface [similar to that in other systems like PTCDA on $\operatorname{Ag}(111),{ }^{7}$ naphthalene-1,4,5,8-tetracaboxylic-dianhydride (NTCDA) on $\mathrm{Ag}(111),{ }^{41}$ or different MePc molecules on $\left.\operatorname{Ag}(111)^{15,16}\right]$. Due to the very broad shape of the F-LUMO peak, no shifting can be unambiguously identified when the coverage is changed. For the HOMO state also a small change in binding energy of $(40 \pm 28) \mathrm{meV}$ was detected. This shift is in the same order of magnitude as the one found for $\mathrm{CuPc}$ on $\mathrm{Ag}(111),{ }^{15}$ and significantly smaller than that on $\mathrm{Au}(111)$. For coverages above $1.00 \mathrm{ML}$ the binding energy of the HOMO state increases from 1.46 to $1.59 \mathrm{eV}$ (at $1.30 \mathrm{ML}$ ). This shift of the HOMO state is caused by a weaker coupling of the photoelectron hole to the metallic states due to the underlying molecular layer.

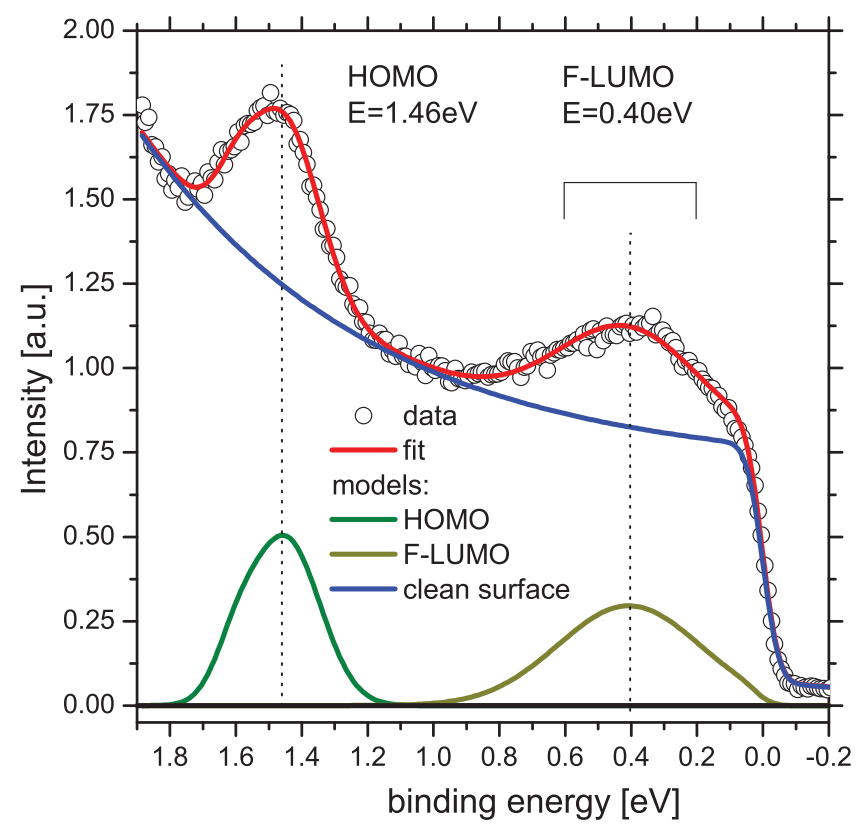

FIG. 10. (Color online) UPS spectra of $\mathrm{CuPc}$ on $\mathrm{Cu}(111)$ for a coverage of $0.85 \mathrm{ML}$, an emission angle of $45^{\circ}$, and $T=-140^{\circ} \mathrm{C}$. 


\section{CONCLUSION}

In the present work and in Ref. 15 the submonolayer adsorption of $\mathrm{CuPc}$ on $\mathrm{Au}(111), \mathrm{Ag}(111)$, and $\mathrm{Cu}(111)$ was investigated comprehensively. All three phase diagrams contain disordered phases at low coverages and long-rangeordered phases at higher coverages. However, even though there seem to be many similarities on first sight, the three systems differ strongly in the interaction strengths between adsorbate molecules and the surface, and consequently the structure formation in the adlayer is fundamentally different. This is most obvious in the high-coverage regime ( $\gtrsim 0.9 \mathrm{ML})$ where point-on-line structures are found on $\mathrm{Au}(111)$ and $\mathrm{Ag}(111)$, whereas on $\mathrm{Cu}(111)$ the molecules grow in commensurate islands.

On $\operatorname{Ag}(111)$ the structural parameters of the molecular layer still depend strongly on the coverage, i.e., the optimum structure corresponding to the minimum total interface potential depends on the coverage. This is caused by adsorbing more molecules in the first layer and maximizing the overlap between the molecular orbitals and the substrate states. The latter leads to the repulsive intermolecular interaction reported by Stadler et $a l .{ }^{14}$ and Kröger et $a l .{ }^{15}$ It clearly demonstrates that the substrate structure influences and restricts the molecular order in the overlayer. In contrast, on $\mathrm{Au}(111)$ there is almost no influence of the changing coverage on the structure of the molecular layer, indicating that only the adsorption of additional molecules yields some energy gain. Only very close to the one-layer coverage does a discrete phase transition occur due to sterical reasons. Hence, the effect of the substrate on the structure formation on the molecular layer is comparatively small. The $\mathrm{Cu}(111)$ surface represents the other extreme. The molecules are strongly chemisorbed and hence the influence of the underlying surface on the molecular growth is strong. The molecules are trapped in commensurate positions on the surface and therefore the layer grows in commensurate islands.

At lower coverages the molecules behave like a 2D gas on $\mathrm{Au}(111)$ and on $\mathrm{Ag}(111)$. From the experimental findings no significant differences were detected for the two substrates. Since for $\mathrm{CuPc} / \mathrm{Au}(111)$ also no clear indication for intermolecular repulsion or attraction was found, we conclude that the disorder in the diluted phases is most likely caused by entropy, an effect which could also not be excluded for $\mathrm{CuPc} / \mathrm{Ag}(111) .{ }^{14,15} \mathrm{On} \mathrm{Cu}(111)$, however, the molecules behave differently. In the diluted disordered regime clear indications for clustering were already found at a coverage of $0.50 \mathrm{ML}$, linear chain formation seen in STM suggests even smaller values. ${ }^{17}$ This indicates that intermolecular attraction is already dominant in this coverage regime and limits molecular diffusion, in strong contrast to the other substrates where thermally activated diffusion causes the gaslike behavior.

The different structure formation caused by different intermolecular interactions can be correlated directly to the differences in the interaction strength between molecules and substrate. For a weak binding on $\mathrm{Au}(111)$ (physisorption) with no visible charge transfer the molecules show no distinct intermolecular interaction. In the case of the stronger binding of the molecules on $\mathrm{Ag}(111)$ (weak chemisorption) the repulsive interaction dominates the structure formation as discussed above, and for even stronger binding on $\mathrm{Cu}(111)$ (strong chemisorption) the molecules show an overall attractive intermolecular interaction. Both attractive and repulsive interactions are caused by the charge exchange between the molecule and the surface and the charge redistribution in the molecule. Since on $\operatorname{Ag}(111)$ the competition of neighboring molecules for charge exchange with the substrate causes a repulsive interaction, one might expect that this effect would become even stronger for the adsorption on $\mathrm{Cu}(111)$ because of the increased charge transfer on this surface. However, the strong interaction with the $\mathrm{Cu}(111)$ surface, in combination with different symmetries of molecule and substrate, breaks the symmetry of the LUMO state and induces a quadrupole moment that is responsible for molecular attraction which in turn overcompensates the repulsion. This leads to the overall attractive intermolecular interaction of $\mathrm{CuPc} / \mathrm{Cu}(111)$ in all coverage regimes. A tendency to a similar behavior was also found for $\mathrm{SnPc}$ on $\mathrm{Ag}(111)$ at very low coverages and low temperatures. ${ }^{42}$ In contrast to $\mathrm{CuPc}$ the SnPc molecule is not planar; consequently it can adsorb in two orientations on the surface: with the Sn atom pointing toward the surface ("Sn down") or into vacuum ("Sn up"). STM showed that at LT and low coverages Sn-up molecules are isolated on the $\operatorname{Ag}(111)$ surface while Sn-down molecules form linear chains. ${ }^{42}$ The reason is that Sn-down molecules interact more strongly with the surface than Sn-up molecules, which (for low coverage and low temperature) causes the same symmetry-breaking effect as discussed for $\mathrm{CuPc} / \mathrm{Cu}(111)$, but only for Sn-down molecules. For Sn-up molecules repulsion remains dominant. The different interaction is caused by a different spatial overlap of molecular states (the HOMO-1 and the LUMO state in particular) of Sn-down and Sn-up molecules.

In conclusion, we want to accentuate that the adsorption of MePcs on noble metal surfaces represents an exceptional model system for studying almost all variations of moleculemolecule and molecule-substrate interactions. By selecting molecules and substrate materials properly the system can be arbitrarily tuned from weak physisorption to strong chemisorption and from attractive to repulsive intermolecular interaction.

\section{SUMMARY}

The submonolayer growth of $\mathrm{CuPc}$ on $\mathrm{Au}(111)$ and $\mathrm{Cu}(111)$ was investigated using high-resolution low-energy electron diffraction and photoelectron spectroscopy. Special emphasis was put on the comparison of the results with the reference system $\mathrm{CuPc} / \mathrm{Ag}(111) .{ }^{15} \mathrm{On} \mathrm{Au}(111)$ the molecules show no long-range order for low coverages, whereas above $0.93 \mathrm{ML}$ point-on-line structures with slightly decreasing area per molecule are observed. The monolayer phase itself turns out to be a mixture of a point-on-line and an incommensurate structure. No clear indications for either repulsive or attractive intermolecular interaction were observed. On $\mathrm{Cu}(111)$, however, the alignment of the molecules indicates an attractive intermolecular overall interaction at all coverages below 1.00 ML (except at very small coverages, where the limited diffusion length inhibits the interaction). Up to $0.76 \mathrm{ML}$ the molecules align in linear chains of different lengths. At higher coverages they start to form islands with 
commensurate structure and two molecules per unit cell. UPS measurements show that the molecular LUMO is now partly filled and shifted below the Fermi edge indicating charge transfer between the molecules and the substrate. No such state was observed on $\mathrm{Au}(111)$. The differences in intermolecular interactions can be correlated with the binding strength of the molecule to the surface: Weakly interacting systems like $\mathrm{CuPc} / \mathrm{Au}(111)$ show no charge transfer and interact only by van der Waals-like forces. A small charge transfer caused by a weak chemical interaction between molecule and surface as for CuPc on $\mathrm{Ag}$ (111) (Ref. 15) leads to a repulsive intermolecular interaction which was explained using a donation-backdonation model. ${ }^{14}$ In strongly binding systems [CuPc on $\mathrm{Cu}(111)]$ this effect is enhanced and results in an even stronger repulsive interaction. However, the larger charge transfer also induces some charge redistribution in the molecule, causing an electrostatic moment. This gives rise to an attractive intermolecular interaction which supersedes the repulsion and leads to chain (and at higher coverages also island) formation of $\mathrm{CuPc}$ on $\mathrm{Cu}(111)$.

\section{ACKNOWLEDGMENTS}

We thank A. Schöll, J. Ziroff and M. Häming for stimulating discussions and acknowledge financial support from the Deutsche Forschungsgemeinschaft (Grant No. KU 1531/2-1 and Graduiertenkolleg GK 1221) and the Bundesministerium für Bildung und Forschung (BMBF Grant No. 03SF0356B GREKOS). *b.stadtmueller@fz-juelich

${ }^{1}$ J. Kido, M. Kimura, and K. Nagai, Science 267, 1332 (1995).

${ }^{2}$ D. Dimitrakopoulos and P. Malenfant, Adv. Mater. 14, 99 (2002).

${ }^{3}$ D. Wöhrle and D. Meissner, Adv. Mater. 3, 129 (1991).

${ }^{4}$ L. Kilian et al., Phys. Rev. Lett. 100, 136103 (2008).

${ }^{5}$ L. Kilian, E. Umbach, and M. Sokolowski, Surf. Sci. 600, 2633 (2006).

${ }^{6}$ L. Kilian, E. Umbach, and M. Sokolowski, Surf. Sci. 573, 359 (2004).

${ }^{7}$ Y. Zou, L. Kilian, A. Schoell, T. Schmidt, R. Fink, and E. Umbach, Surf. Sc. 600, 1240 (2006).

${ }^{8}$ L. Romaner, D. Nabok, P. Puschnig, E. Zojer, and C. AmbroschDraxl, New J. Phys. 11, 053010 (2009).

${ }^{9}$ S. Duhm, A. Gerlach, I. Salzmann, B. Bröker, R. L. Johnson, F. Schreiber, and N. Koch, Org. Electron. 9, 111 (2008).

${ }^{10}$ F. Tautz, Prog. Surf. Sci. 82, 479 (2007) .

${ }^{11}$ T. Wagner, A. Bannani, C. Bobisch, H. Karacuban, and R. Möller, Condens. Matter Phys. 19, 056009 (2007).

${ }^{12}$ C. Stadler, S. Hansen, A. Schöll, T.-L. Lee, J. Zegenhagen, C. Kumpf, and E. Umbach, New J. Phys. 9, 50 (2007).

${ }^{13}$ J. Stanzel, W. Weigand, L. Kilian, H. Meyerheim, C. Kumpf, and E. Umbach, Surf. Sci. 571, 1 (2004).

${ }^{14}$ C. Stadler, S. Hansen, I. Kröger, C. Kumpf, and E. Umbach, Nature Phys. 5, 153 (2009).

${ }^{15}$ I. Kröger et al., New J. Phys. 12, 083038 (2010).

${ }^{16}$ M. Häming, C. Scheuermann, A. Schöll, F. Reinert, and E. Umbach, in Advanced Spectroscopies of Molecular Materials for Electronics, edited by N. Ueno, M. Fahlman and N. Koch, special issue of J. Electron Spectrosc. Relat. Phenom. 174, 59 (2009).

${ }^{17}$ H. Karacuban, M. Lange, J. Schaffert, O. Weingart, T. Wagner, and R. Möller, Surf. Sci. 603, L39 (2009).

${ }^{18}$ S. C. B. Mannsfeld and T. Fritz, Phys. Rev. B 71, 235405 (2005).

${ }^{19}$ C. Stadler, S. Hansen, F. Pollinger, C. Kumpf, E. Umbach, T.-L. Lee, and J. Zegenhagen, Phys. Rev. B 74, 035404 (2006).

${ }^{20}$ J. Buchholz and G. Somorjai, J. Chem. Phys. 66, 573 (1977).

${ }^{21}$ I. Chizhov, G. Scoles, and A. Kahn, Langmuir 16, 4358 (2000).

${ }^{22}$ C. Wagner, D. Kasemann, C. Golnik, R. Forker, M. Esslinger, K. Müllen, and T. Fritz, Phys. Rev. B 81, 035423 (2010).

${ }^{23}$ M. V. Hove, R. Koestner, P. Stair, J. Bibrian, L. Kesmodel, I. Bartoš, and G. Somorjai, Surf. Sci. 103, 189 (1981).
${ }^{24}$ J. V. Barth, H. Brune, G. Ertl, and R. J. Behm, Phys. Rev. B 42, 9307 (1990).

${ }^{25}$ C. Wöll, S. Chiang, R. J. Wilson, and P. H. Lippel, Phys. Rev. B 39, 7988 (1989).

${ }^{26}$ V. Zielasek, A. Büssenschütt, and M. Henzler, Phys. Rev. B 55, 5398 (1997).

${ }^{27}$ J. T. McKinney, E. R. Jones, and M. B. Webb, Phys. Rev. 160, 523 (1967).

${ }^{28}$ Z. H. Cheng, L. Gao, Z. T. Deng, N. Jiang, Q. Liu, D. X. Shi, S. X. Du, H. M. Guo, and H.-J. Gao, J. Phys. Chem. C 111, 9240 (2007).

${ }^{29}$ P. H. Lippel, R. J. Wilson, M. D. Miller, C. Wöll, and S. Chiang, Phys. Rev. Lett. 62, 171 (1989).

${ }^{30}$ D. Hooks, T. Fritz, and M. Ward, Adv. Mater. 13, 227 (2001).

${ }^{31}$ A. Hoshino, S. Isoda, H. Kurata, and T. Kobayashi, J. Appl. Phys. 76, 4113 (1994).

${ }^{32}$ S. Mannsfeld, M. Toerker, T. Schmitz-Hübsch, F. Sellam, T. Fritz, and K. Leo, Org. Electron. 2, 121 (2001).

${ }^{33}$ J. K. Gimzewski, S. Modesti, C. Gerber, and R. R. Schlittler, Chem. Phys. Lett. 213, 401 (1993).

${ }^{34}$ F. Sellam, T. Schmitz-Höbsch, M. Toerker, S. Mannsfeld, H. Pröhl, T. Fritz, K. Leo, C. Simpson, and K. Möllen, Surf. Sci. 478, 113 (2001).

${ }^{35}$ J. Ziroff, F. Forster, A. Schöll, P. Puschnig, and F. Reinert, Phys. Rev. Lett. 104, 233004 (2010).

${ }^{36}$ F. Evangelista, V. Carravetta, G. Stefani, B. Jansik, M. Alagia, S. Stranges, and A. Ruocco, J. Chem. Phys. 126, 124709 (10) (2007).

${ }^{37}$ T. Kataoka, H. Fukagawa, S. Hosoumi, K. Nebashi, K. Sakamoto, and N. Ueno, Chem. Phys. Lett. 451, 43 (2008).

${ }^{38}$ T.-C. Chiang, G. Kaindl, and T. Mandel, Phys. Rev. B 33, 695 (1986).

${ }^{39}$ M. Horn and von Hoegen, Z. Kristallogr. 214, 1 (1999).

${ }^{40}$ K. Gronwald and M. Henzler, Surf. Sci. 117, 180 (1982).

${ }^{41}$ A. Bendounan, F. Forster, A. Schoell, D. Batchelor, J. Ziroff, E. Umbach, and F. Reinert, in ECOSS-24, Proceedings of the 24th European Conference on Surface Science, edited by Jacques Jupille and Paul Dumas [Surf. Sci. 601, 4013 (2007)].

${ }^{42}$ Y. Wang, J. Kröger, R. Berndt, and W. Hofer, Angew. Chem. 121, 1287 (2009). 